\title{
Quantum key distribution with 2-bit quantum codes
}

\author{
Xiang-Bin Wang* \\ Imai Quantum Computation and Information project, ERATO, Japan Sci. and Tech. Corp. \\ Daini Hongo White Bldg. 201, 5-28-3, Hongo, Bunkyo, Tokyo 113-0033, Japan
}

We propose a prepare-and-measure scheme for quantum key distribution with 2-bit quantum codes. The protocol is unconditionally secure under whatever type of intercept-andresend attack. Given the symmetric and independent errors to the transmitted qubits, our scheme can tolerate a bit error rate up to $26 \%$ in 4 -state protocol and $30 \%$ in 6 -state protocol, respectively. These values are higher than all currently known threshold values for the prepare-and-measure protocols. Moreover, we give a practically implementable linear optics realization for our scheme.

Introduction. Quantum key distribution (QKD) is different from classical cryptography in that an unknown quantum state is in principle not known unless it is disturbed, rather than the conjectured difficulty of computing certain functions. The first published protocol, proposed in 1984 [1], is called BB84 (C. H. Bennett and G. Brassard.) For a history of the subject, one may see e.g. [2]. Since then, studies on QKD are extensive. Strict mathematical proofs for the unconditional security have been given already [3-5]. It is greatly simplified if one connects this with the quantum entanglement purification protocol (EPP) [3,6-10]. Very recently, motivated for higher bit error rate tolerance and higher efficiency, Gottesman and Lo [11] studied the classicalization of EPP with two way communications (2-EPP). Their protocol has increased the tolerable bit error rate of channel to $18.9 \%$ and $26.4 \%$ for 4-state QKD and 6-state QKD, respectively. Very recently, these values have been upgraded to $20 \%$ and $27.4 \%$ by Chau [12].

The type of prepare-and-measure QKD schemes is particularly interesting because it does not need the very difficult technique of quantum storage. In this paper, we propose a new prepare-and-measure scheme with the assistance of 2-bit quantum codes. The linear optical realization is shown in Fig. $(1,2)$. In our scheme, Alice shall send both qubits of the quantum codes to Bob, therefore they do not need any quantum storage. Bob will first check the parity of the two qubits by the polarizing beam splitter (PBS) and then decode the code with post selection. The 2-bit code is produced by the SPDC process [13], see in Fig.(1).

We shall use the representation of $|0\rangle=\left(\begin{array}{l}1 \\ 0\end{array}\right) ;|1\rangle=$ $\left(\begin{array}{l}0 \\ 1\end{array}\right)$. We denote $\sigma_{x}=\left(\begin{array}{ll}0 & 1 \\ 1 & 0\end{array}\right), \sigma_{z}=\left(\begin{array}{cc}1 & 0 \\ 0 & -1\end{array}\right), \sigma_{y}=$

*email: wang@qci.jst.go.jp $\left(\begin{array}{cc}0 & -i \\ i & 0\end{array}\right)$. These operators represent for a bit flip error only, a phase flip error only and both error, respectively. The detected bit (or phase) flip error rate is the summation of $\sigma_{x}$ (or $\sigma_{z}$ ) error rate and $\sigma_{y}$ error rate. The $\mathrm{Z}, \mathrm{X}, \mathrm{Y}$ basis are defined by the basis of $\{|0\rangle,|1\rangle\},\{|0\rangle \pm|1\rangle\},\{|0\rangle \pm i|1\rangle\}$, respectively.

Main idea. We propose a revised 2-EPP scheme which is unconditionally secure and which can further increase the thresholds of error rates given the independent channel errors. We propose to let Alice send Bob the quantum states randomly chosen from $\left\{\frac{1}{\sqrt{2}}(|00\rangle+|11\rangle), \frac{1}{\sqrt{2}}(|00\rangle-\right.$ $|11\rangle),|00\rangle,|11\rangle\}$. As we shall see, these states are just the quantum phase-flip error-rejection (QPFER) code for the BB84 state $\left\{|0\rangle,|1\rangle, \frac{1}{\sqrt{2}}(|0\rangle+|1\rangle), \frac{1}{\sqrt{2}}(|0\rangle-|1\rangle)\right\}$.

In our 4-state protocol, the tolerable channel bit-flip and phase-flip rate is raised to $26 \%$ for the symmetric channel with independent noise. (A symmetric channel is defined as the one with equal distribution of errors of $\sigma_{x}, \sigma_{z}, \sigma_{y}$.) Note that the theoretical upper bound of $25 \%$ [11] only holds for those 4-state schemes where Alice and Bob only test the error rate before any error removing steps. However, this is not true with the delay of error test. Considering the standard purification protocol [7] with symmetric channel, one may distill the maximally entangled states out of the raw pairs whose initial bit-flip error and phase-flip error are $33.3 \%$. In our 4-state protocol, we delay the error test by one step of purification with 2-bit QPFER code. This raises the tolerable channel flipping rates.

The QPFER code. We shall use the following QPFER code:

$$
\begin{gathered}
|0\rangle|0\rangle \longrightarrow(|00\rangle+|11\rangle) / \sqrt{2} \\
|1\rangle|0\rangle \longrightarrow(|00\rangle-|11\rangle) / \sqrt{2}
\end{gathered}
$$

Here the second qubit in the left side of the arrow is the ancilla for the encoding. This code is not assumed to reduce the errors in all cases. But in the case that the channel noise is uncorrelated or nearly uncorrelated, it works effectively. Consider an arbitrary state $\alpha|0\rangle_{1}+$ $\beta|1\rangle_{1}$ (qubit 1) and an ancilla state $|0\rangle_{2}$ (qubit 2). Taking unitary transformation of $\mathrm{Eq}(1)$ we obtain the following un-normalized state:

$$
\alpha\left(|0\rangle_{1}|0\rangle_{2}+|1\rangle_{1}|1\rangle_{2}\right)+\beta\left(|0\rangle_{1}|0\rangle_{2}-|1\rangle_{1}|1\rangle_{2}\right)
$$

This can be regarded as the encoded state for $\alpha|0\rangle_{1}+$ $\beta|1\rangle_{1}$. Alice then sends both qubits to Bob. In receiving them, Bob first takes a parity check, i.e. he compares the bit values of the two qubits in $\mathrm{Z}$ basis. Note that 
this collective measurement does not destroy the code state itself. Specifically, the parity check operation can be done by the PBS in Fig.(2): there, states $|0\rangle,|1\rangle$ are for horizontal and vertical polarization photon states, respectively. Since a PBS transmits $|0\rangle$ and reflects $|1\rangle$, if incident beams (beam 1 and 2) of the PBS are both horizontally polarized or vertically polarized, there must be one photon on each output beams (beam 1' and 2'); if the polarizations of two incident beams are one horizontal and one vertical, one of the output beams must be empty. After the parity check, if bit values are different, Bob discards the whole 2-qubit code, if they are same, Bob decodes the code. In decoding, he measures qubit 1 in $X$ basis, if he obtains $|+\rangle$, he takes a Hadamard transformation $H=\frac{1}{\sqrt{2}}\left(\begin{array}{cc}1 & 1 \\ 1 & -1\end{array}\right)$ to qubit 2 ; if he obtains $|-\rangle$ for qubit 1, he takes the Hadamard transformation to qubit 2 and then flips qubit 2 in Z basis. Suppose the original channel error rates of $\sigma_{x}, \sigma_{y}, \sigma_{z}$ types are $p_{x 0}, p_{y 0}, p_{z 0}$, respectively. Let $p_{I 0}=1-p_{x 0}-p_{y 0}-p_{z 0}$. One may easily verify the probability distribution and error type for the survived and decoded states (qubit 2) in following table

\begin{tabular}{r|r|r|r}
\hline JCE & probability & decoded state & error type \\
\hline$I \otimes I$ & $p_{I 0}^{2}$ & $\alpha|0\rangle+\beta|1\rangle$ & $I$ \\
\hline$\left\{I \otimes \sigma_{z}\right\}$ & $2 p_{I 0} p_{z 0}$ & $\alpha|1\rangle+\beta|0\rangle$ & $\sigma_{x}$ \\
\hline$\sigma_{z} \otimes \sigma_{z}$ & $p_{z 0}^{2}$ & $\alpha|0\rangle+\beta|1\rangle$ & $I$ \\
\hline$\sigma_{y} \otimes \sigma_{y}$ & $p_{y 0}^{2}$ & $\alpha|0\rangle-\beta|1\rangle$ & $\sigma_{z}$ \\
\hline$\sigma_{x} \otimes \sigma_{x}$ & $p_{x 0}^{2}$ & $\alpha|0\rangle-\beta|1\rangle$ & $\sigma_{z}$ \\
\hline$\left\{\sigma_{x} \otimes \sigma_{y}\right\}$ & $2 p_{x 0} p_{y 0}$ & $\alpha|1\rangle-\beta|0\rangle$ & $\sigma_{y}$
\end{tabular}

The first column lists the various types of joint channel errors(JCE) before decoding. $\{\alpha \otimes \beta\}$ denotes both $\alpha \otimes$ $\beta$ and $\beta \otimes \alpha$. According to this table, the error rate distribution for the survived raw pairs after decoding is:

$$
\left\{\begin{array}{l}
p_{I}=\frac{p_{I 0}^{2}+p_{z 0}^{2}}{\left(p_{I 0}+p_{z 0}\right)^{2}+\left(p_{x 0}+p_{y 0}\right)^{2}}, \\
p_{z}=\frac{p_{x 0}^{2}+p_{y 0}^{2}}{\left(p_{I 0}+p_{z 0}\right)^{2}+\left(p_{x 0}+p_{y 0}\right)^{2}}, \\
p_{y}=\frac{2 p_{x 0} p_{y 0}}{\left(p_{I 0}+p_{z 0}\right)^{2}+\left(p_{x 0}+p_{y 0}\right)^{2}}, \\
p_{x}=\frac{2 p_{I 0} p_{z 0}}{\left(p_{I 0}+p_{z 0}\right)^{2}+\left(p_{x 0}+p_{y 0}\right)^{2}}
\end{array}\right.
$$

With this formula, the phase flip error to the decoded states is obviously reduced. Note that this formula does not hold for the correlated channel errors. Even though the noise of the physical channel is uncorrelated, in carrying out the QKD task, we should not use this formula to deduce the flipping rates of the decoded qubits based on our knowledge of the physical channel noise, i.e., the values of $p_{I 0}, p_{x 0}, p_{y 0}, p_{z 0}$. But we can choose to directly test the error rate of the survived and decoded qubits and to see whether formula (3) indeed holds, based on our prior knowledge of physical channel noise.

Our protocol with linear optical realization. In the BB84 protocol, there are only four different states. Therefore Alice may directly prepare random states from the set of $\left\{\frac{1}{\sqrt{2}}(|00\rangle+|11\rangle), \frac{1}{\sqrt{2}}(|00\rangle-|11\rangle),|00\rangle,|11\rangle\right\}$ and sends them to Bob. This is equivalent to first preparing the BB84 states and then encoding them by eq.(1). We propose the following 4-state protocol with implementation of linear optics in Fig(1) and Fig(2):

1 Alice prepares N 2-qubit quantum codes with $N / 4$ of them being prepared in $|00\rangle$ or $|11\rangle$ with equal probability and $3 N / 4$ of them being prepared in $\frac{1}{\sqrt{2}}(|00\rangle \pm|11\rangle)$ with equal probability. All codes are put in randomly order. She records the the "preparation basis" as X basis for code $|00\rangle$ or $|11\rangle$; and as $\mathrm{Z}$ basis for code $\frac{1}{\sqrt{2}}(|00\rangle \pm|11\rangle)$. And she records the bit value of 0 for the code $|00\rangle$ or $\frac{1}{\sqrt{2}}(|00\rangle+|11\rangle)$; bit value 1 for the code $|11\rangle$ or $\frac{1}{\sqrt{2}}(|00\rangle-|11\rangle)$. She sends each 2-qubit code to Bob. In Fig.(1), any of the above four states can be produced from the nonlinear crystal by appropriately setting the polarization of the pump light [15]. 2 Bob checks the parity of each 2-qubit code in $\mathrm{Z}$ basis. He discards the codes whenever the 2 bits have different values and he takes the following measurement if they have same values: he measures qubit 1 in $\mathrm{X}$ basis and qubit 2 in either $\mathrm{X}$ basis or $\mathrm{Z}$ basis with equal probability. If Bob has measured qubit 2 in $\mathrm{X}$ (or ) Z basis, he records the "measurement basis" as Z (or ) X basis [16] and we shall simply call the qubit as Z-bit (or X-bit) latter on. If he obtains $|+\rangle|+\rangle$, $|+\rangle|0\rangle,|-\rangle|-\rangle$ or $|-\rangle|0\rangle$, he records bit value 0 for that code; if he obtains $|-\rangle|+\rangle,|-\rangle|1\rangle,|+\rangle|-\rangle$ or $|+\rangle|1\rangle$, he records bit value 1 for that code. In our linear optical realization, Bob's detections are done by post selection in Fig.(2): If beam 1' and beam 2' each contains one photon, beam 1 and beam 2 must have the same bit values. Otherwise, their bit values must be different. This requires Bob to only accept the events of two fold clicking with one clicked detector from $\{\mathrm{D} 1, \mathrm{D} 2\}$ and the other clicked detector from $\{\mathrm{D} 3, \mathrm{D} 4, \mathrm{D} 5, \mathrm{D} 6\}$. All other types of events must be discarded. Moreover, according to the above mentioned corresponding rule, to those accepted events, clicking of D3 or D4 means measurement in Z basis to beam 2', corresponding to "X basis" for his record; also, clicking of D5 or D6 means measurement in X basis to beam 2', corresponding to "Z basis" for his record. The two fold clicking of (D1,D6),(D1,D3),(D2,D5) or $(\mathrm{D} 2, \mathrm{D} 3)$ corresponds to bit value of 0 ; two fold clicking of (D2,D6),(D2,D4), (D1,D5) or (D1,D3) corresponds to bit value 1. 3 Bob announces which codes have been discarded. Alice and Bob compare the "preparation basis" and "measurement basis" of each bits decoded from the survived codes by classical communication. They discard those bits whose "measurement basis" disagree with "preparation basis". Bob announces the bit value of all X-bits. He also randomly chooses the same number of 
$Z$-bits and announces their values. If too many of them disagree with Alice's record, they abort the protocol. 4 Now they regard the tested error rates on Z-bits as the bit-flip rate and the tested error rate on X-bits as phase flip rate. They reduce the bit flip rate in the following way: they randomly group all their unchecked bits with each group containing 2 bits. They compare the parity of each group. If the results are different, they discard both bits. If the results are same, they discard one bit and keep the other. They repeatedly do so for a number of rounds until they believe that both bit flip rate and phase flip rate can be reduced to less than $5 \%$ with the next step being taken. $\mathbf{5}$ They then randomly group the remained bits with each group containing $r$ bits. They use the parity of each group as the new bits. 6 They use the classical CSS code [6] to distill the final key.

Note that in this protocol, Since formula (3) is not unconditionally true, Alice and Bob check the bit errors after decoding the 2-bit quantum codes. If the detected errors are significantly larger than the expected values calculated from eq.(3), they will abort the protocol. That is to say, if formula (3) really works, they continue, if it does not work, they abort it. After any round of bit flip error rejection in step 4, the error rate will be iterated by equation (1) in ref. [12]. After the phase error correction in step 5 , the new error rate satisfies the inequality of formula (3) of ref. [12] provided that $p_{I}>1 / 2$. The above steps to remove the bit-flip error and phase-flip error are unconditionally true since Alice and Bob have paired the qubits randomly. Even though the errors of the decoded qubits are arbitrarily correlated, the above steps always work as theoretically expected.

Given $p_{x}, p_{y}, p_{z}$, if there exits a finite number $k$, after $k$ rounds of bit-flip error-rejection, we can find a $r$ which satisfy

$$
\begin{array}{r}
r\left(p_{x}+p_{y}\right) \leq 5 \% \\
e^{-2 r\left(0.5-p_{z}-p_{y}\right)^{2}} \leq 5 \%,
\end{array}
$$

one can then obtain the unconditionally secure and faithful final key with a classical CSS code [6].

In the 4-state protocol, we don't detect the $\sigma_{y}$ error for the states decoded from the survived codes, therefore we have to assume $p_{y}=0$ after the quantum parity check and decoding. But we do not have to assume $p_{y 0}=0$, actually Alice and Bob never test any error rate before decoding in the protocol. However, if the channel noise is symmetric and uncorrelated, after the quantum decoding, both $\sigma_{z}$ error $\left(p_{z}\right)$ and $\sigma_{y}$ error $\left(p_{y}\right)$ are reduced, i.e., the detectable phase error rate has been reduced in a rate as it should be i.e., eq.(3). We then start from the un-symmetric error rate with assumption $p_{y}=0$ and $p_{x}, p_{z}$ being the detected bit-flip rate and phase-flip rate, respectively. After the calculation, we find that the tolerable error rate of bit flip or phase flip is $26 \%$ for the 4 -state protocol. Moreover, in the case that the channel error distribution itself is $p_{y 0}=0 ; p_{x 0}=p_{z 0}$, the tolerable channel error rate for our protocol is $p_{x 0}=p_{z 0} \leq 21.7 \%$.
The above protocol is totally equivalent to the one based on entanglement purification therefore it is unconditionally secure [14]. Here we give a simple security proof.

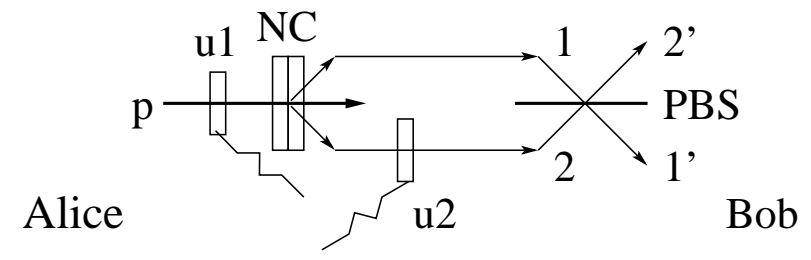

FIG. 1. QKD scheme with 2-bit quantum codes. PBS: polarizing beam splitter. NC: nonlinear crystals used in SPDC process, p: pump light in horizontal polarization, $\mathrm{u} 1$ : unitary rotator, u2: phase shifter. $\mathrm{u} 1$ takes the value of $0, \pi / 2, \pi / 4$ to produce emission state $|11\rangle,|00\rangle,\left|\phi^{+}\right\rangle$, respectively. u2 can be either $I$ or $\sigma_{z}$

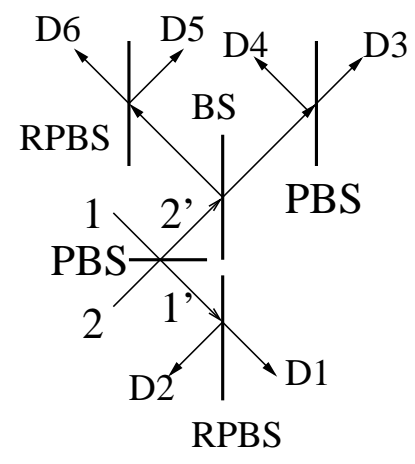

FIG. 2. Bob's action in QKD scheme of figure(1). RPBS: Rotated polarizing beam splitter which transmits the state $|+\rangle$ and reflects state $|-\rangle)$. BS: 50:50 beam splitter. D represents for a photon detector. With RPBS, one may measure the incident beam in $\{|+\rangle,|-\rangle\}$ basis.

Security proof. Consider two protocols, protocol P0 and protocol P. In protocol P0, Alice directly sends Bob each individual qubits. In protocol P, Alice first encodes each individual qubits by a certain error rejection code and then sends each quantum codes to Bob. We denote encoding operation as $\hat{E}$ and parity check and decoding operation by $\hat{D}$. Bob will first check parity of each code and decode the survived codes. After decoding, Alice and Bob continue the protocol. Suppose except for the operations of $\hat{D}$ and $\hat{E}$ everything else in protocol P0 and protocol $\mathrm{P}$ is identical and operation $\hat{D}$ or $\hat{E}$ do not require any information of the original qubit itself, then we have the following theorem: If protocol P0 is secure with arbitrary lossy channel, then protocol $P$ is also secure. The proof of this theorem is very simple. Suppose $\mathrm{P}$ is insecure. Then Eve. must be able to attack the final key by certain operation. Denote Eve's attack during the period that all codes are transmitted from Alice to Bob as $\hat{A}$. Eve may obtain significant information to final key with operation $\hat{A}$ and other operations 
$(\hat{Q})$ after Bob receives the qubits. If this is true, then in protocol P0, Eve may take the operation of $\hat{D} \hat{A} \hat{E}$ in the same period and then send the decoded states to Bob, with all other operations identical to those in protocol $\mathrm{P}$. (The time order is from right to left.) To Alice and Bob, it looks like that they are carrying out protocol P0 with a lossy channel now, because Eve will have to discard some of the 2-bit quantum codes after the parity check in decoding. All final results from protocol $\mathrm{P} 0$ with attack $\hat{Q} \hat{D} \hat{A} \hat{E}$ must be identical to protocol $\mathrm{P}$ with attack $\hat{Q} \hat{A}$, since everything there with the two protocols are now the same. This completes our proof of the theorem. Our QKD protocol in previous section is just the modified Chau protocol [12] with encoding and decoding added. We can regard our protocol as $\mathrm{P}$ and Chau protocol as P0 in applying our theorem. Since Gottesman-Lo protocol [11] or Chau protocol [12] are all unconditionally secure with arbitrary lossy channel, we conclude that our protocol must be also unconditionally secure.

6 -state protocol. Our protocol can obviously be extended to the 6 -state protocol [17]. In doing so, Alice just change the initially random codes by adding $N / 4$ codes from $\left\{\frac{1}{2}[(|00\rangle+|11\rangle) \pm i(|00\rangle-|11\rangle)]\right\}$. This is equivalent to $\frac{1}{\sqrt{2}}(\{|00\rangle \mp i|11\rangle)\}$. She regards all this type codes as Ybits. In decoding the codes, Bob's "measurement basis" is randomly chosen from 3 basis, $\mathrm{X}, \mathrm{Y}$ and $\mathrm{Z}$. All decoded $\mathrm{X}$-bits, Y-bits and the same number of randomly chosen decoded $\mathrm{Z}$ will be used as the check bits. Since the Hadamard transform switches the two eigenstates of $\sigma_{y}$, after decoding, whenever Bob measures qubit 2 in Y basis, he needs to flip the measurement outcome so that to obtain everything the same as that in the 2-EPP with quantum storages [14]. In such a way, if the channel is symmetric, Bob will find $p_{y} \neq 0$. And he will know $p_{x}, p_{y}, p_{z}$ exactly instead of assuming $p_{y}=0$. This will increase the tolerable error rate accordingly. In the case symmetric physical channel, our 6-state protocol tolerates the flipping rate up to $30 \%$.

Subtlety of the "conditional advantage". un-symmetric effective channel. Although the advantage of a higher threshold is conditional, the security of our protocol is unconditional. That is to say, whenever our protocol produces any final key, Eve's information to that key must be exponentially close to zero, no matter whether Eve uses coherent attack or individual attack. Our protocol is totally different from the almost useless protocol which is only secure with uncorrelated channel noise. There are two conditions for the error threshold advantage:

(1) The noise of physical channel should be the type where eq.(3) holds; (2) Eve. is not detected in the error test, i.e., the result of error test must be in agreement with the expected result given by eq.(3).

Both conditions here are verifiable by the protocol itself. The second condition is a condition for any QKD protocol. The first condition is on the known physical channel rather than Eve's channel in QKD. In our protocol, Eve's attack must not affect the error rates detected on the decoded qubits if she wants to hide her presence. That is to say, if Eve hides her presence, all results about the final key of our protocol can be correctly estimated based on the known properties of the physical channel, no matter what type of attack she has used. Given a physical channel with its noise being un-correlated and symmetric and higher than the thresholds of all other prepare-andmeasure protocols but lower than that of our protocol, our protocol is the only one that works. In practice, one may simply separate the 2 qubits of the quantum code substantially to guarantee the un-correlation of the physical channel noise. This is to say, the error threshold advantage of our protocol is actually unconditional in practice. Loose ends in practice. Multi-pair emission in SPDC and dark counting of detectors have not been considered. We believe these issues can be resolved along the similar lines in the case of BB84 implemented with a weak coherent light source.

Acknowledgement: I thank Prof Imai H for support. The extended version of this work has been submitted to QIT-EQIS workshop (Kyoto) [14]. I thank the anonymous referees of the workshop for their comments and suggestions. I thank Dr B. S. Shi for discussions on the source design.

[1] C. H. Bennett and G. Brassard, Proceedings of IEEE International Conference on Computers, Systems and Signal Processing, Bangalore, India, 1984, (IEEE Press, 1984), pp. 175-179; C.H. Bennett and G. Brassard, IBM Technical Disclosure Bulletin 28, 3153-3163 (1985).

[2] N. Gisin, G. Ribordy, W. Tittel, and H. Zbinden, Reviews of Modern Physics, vol. 74, pp. 145-195.

[3] H.-K. Lo and H. F. Chau, Science, 283, 2050(1999)

[4] D. Mayers, Journal of ACM, 48,351.

[5] E. Biham, M. Boyer, P. O. Boykin, T. Mor, and V. Roychowdhury, Proceedings of the Thirty-Second Annual ACM Symposium on Theory of Computing (STOC) (ACM Press, New York, 2000), p. 715.

[6] P. W. Shor and J. Preskill, Phys. Rev. Lett., $85,441(2000)$.

[7] C. H. Bennett, D. P. DiVincenzo, J. A. Smolin, and W. K. Wootters, Phys. Rev. A54, 3824(1996)

[8] D. Deutsch, A. Ekert, R. Jozsa, C. Macchiavello, S. Popescu, and A. Sanpera, Phys. Rev. Lett., 77, 2818(1996); Erratum Phys. Rev. Lett. 80, 2022 (1998).

[9] D. Gottesman and J. Preskill, Phys. Rev.A63,22309(2001).

[10] A. R. Calderbank and P. Shor, Phys. Rev. A54, 1098(1996), A. M. Steane, Proc. R. Soc. London A452, 2551(1996).

[11] D. Gottesman and H.-K. Lo, IEEE Transactions on Information Theory, 49, 457(2003).

[12] H. F. Chau, Phys. Rev. A66, 060302(R) (2002). 
[13] P. G. Kwiat, K. Mattle, H. Weinfurter, A. Zeilinger, A.V. Sergienko, and Y. H. Shih, Phys. Rev. Lett. 75, 4337(1995).

[14] One may see details in X.B. Wang, quant-ph/0306156.

[15] P.G. Kwiat et al, Phys. Rev. A60, R773(1999).

[16] Here Bob has omitted the Hadamard transformation as appeared in the EPP.

[17] D. Bruss, Phys. Rev. Lett. 81, 3018(1998). 